\title{
Crystallization of weakly charged colloidal spheres: a numerical study
}

\author{
Stefan Auer and Daan Frenkel \\ FOM Institute for Atomic and Molecular Physics, Kruislaan 407, 1098 SJ Amsterdam, \\ The Netherlands
}

Received 17 June 2002

Published 9 August 2002

Online at stacks.iop.org/JPhysCM/14/7667

\begin{abstract}
We report a numerical study of crystal nucleation in a system of weakly charged colloids. The interaction between the colloids is approximated by a repulsive hard-core Yukawa potential. We studied the dependence of the nucleation barrier and the nucleation rate on supersaturation as a function of both contact value and range of the interaction potential. We find that, at the same volume fraction, nucleation is much faster for these soft colloids than for hard spheres. This is partly because fluid-solid coexistence in charged colloids occurs at lower volume fractions than for hard spheres. But, in addition, the softness of the potential has a pronounced direct effect on the nucleation barrier through a lowering of the solid-liquid surface free energy. Moreover, the softness of the potential directly affects the pathway for crystal nucleation: even when the stable crystal phase has a face-centred cubic structure, we find that the initial crystal nuclei have a bcc structure.
\end{abstract}

\section{Introduction}

When a suspension of monodisperse colloids is brought to a sufficiently high density, it will form beautiful, iridescent crystals. The rate at which these crystals form depends strongly on the steepness of the repulsive forces that act between the colloids. While it is difficult to compare absolute nucleation rates in different colloidal systems under differing conditions, experiments $[1,2]$ clearly indicate that charged colloids with a soft, long-ranged repulsion tend to crystallize much faster than hard-sphere colloids at the same supersaturation. The aim of the present paper is to elucidate the factors that affect the rate of crystal nucleation in a system of weakly charged colloids.

In suspension, the charged colloids are surrounded by a cloud of counter-ions. This counter-ion double layer screens the pure Coulomb interaction between the colloids. If we use the linearized Poisson-Boltzmann equation to describe the charge distribution around a charged colloid with hard-core diameter $\sigma$, then we obtain the following expression for the pair interaction between two charged macro-ions: 


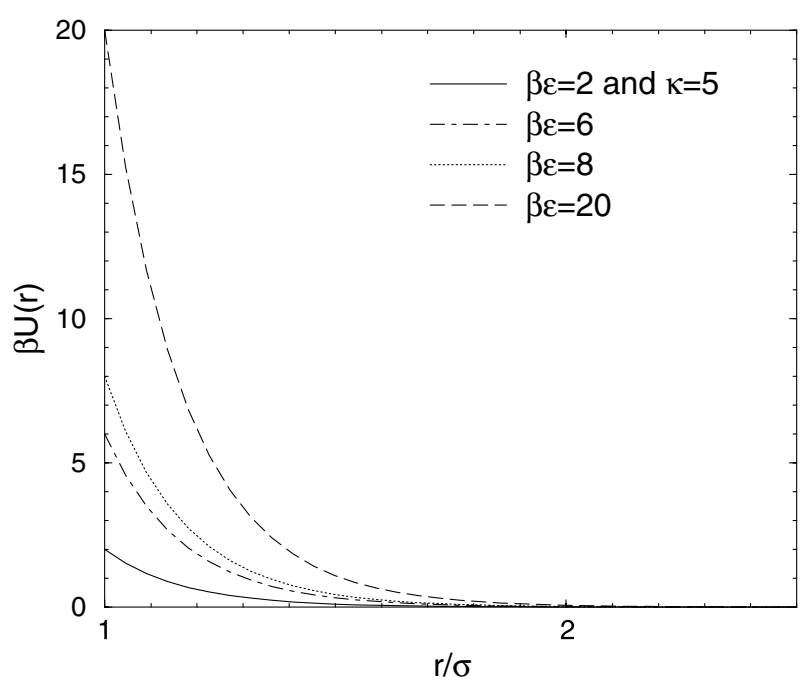

Figure 1. A plot of the hard-core Yukawa potential for $\kappa=5$ as a function of the Yukawa repulsion $\beta \epsilon=2,6,8,20$.

$$
\beta U(r)= \begin{cases}\infty & \text { for } r<\sigma \\ \beta \epsilon \frac{\exp (-\kappa(r / \sigma-1))}{r / \sigma} & \text { for } r>\sigma\end{cases}
$$

$U(r)$ is usually referred to as the 'hard-core Yukawa potential'; see figure 1. Here $\kappa$ is the inverse screening length in units of the hard-sphere diameter $\sigma$ and $\beta \epsilon$ is the value of the Yukawa repulsion at contact. $\beta$ is a measure for the inverse temperature $\left(\beta=1 / k_{B} T\right)$, where $k_{B}$ is the Boltzmann constant. In the linearized Poisson-Boltzmann theory, we have explicit expressions for both $\kappa$ and $\epsilon$ in terms of the size and surface charge of the colloid, and of the concentration of counter-ions and added salt. However, the linearized Poisson-Boltzmann description provides only an approximation to the real colloid-colloid interaction. For instance, it is expected to break down at short distances and for low added salt concentrations. A way to treat the interaction between charged colloids at short distances was already proposed by Derjaguin, Landau, Verweij and Overbeek (DLVO) in the 1940s [3]. Since then, several modifications of the form of the pair potential between charged colloids have been proposed $[4,5]$ but, except at very short distances, most expression are very similar to the hard-core Yukawa model. The main difference between the theories is in the values that they yield for $\kappa$ and $\epsilon$. In the original DLVO theory, these parameters depend only on the ionic strength of the solution and on the bare charge of the colloids. In the more recent theories, $\kappa$ and $\epsilon$ may themselves depend on the concentration of charged colloids. In the present work, we simply assume that the interaction between charged colloids is adequately described by a hard-core Yukawa potential. However, we shall return later to the question of whether this is allowed. A special case of the hard-core Yukawa model, is the hard-sphere model. The latter model applies in the limit of high salt concentrations $\kappa \rightarrow \infty$ and in the limit where the strength of the repulsion is much less than the thermal energy, i.e. $\beta \epsilon \rightarrow 0$. This is typically the case for weakly charged colloids. We note that, whilst the hard-core Yukawa model is commonly used to describe slightly charged colloids, it can also be used as a crude model for sterically stabilized colloids. Hence, many of the conclusions that we obtain below, in particular those for systems with a high value of $\kappa$, should equally apply to sterically stabilized, uncharged colloids. 


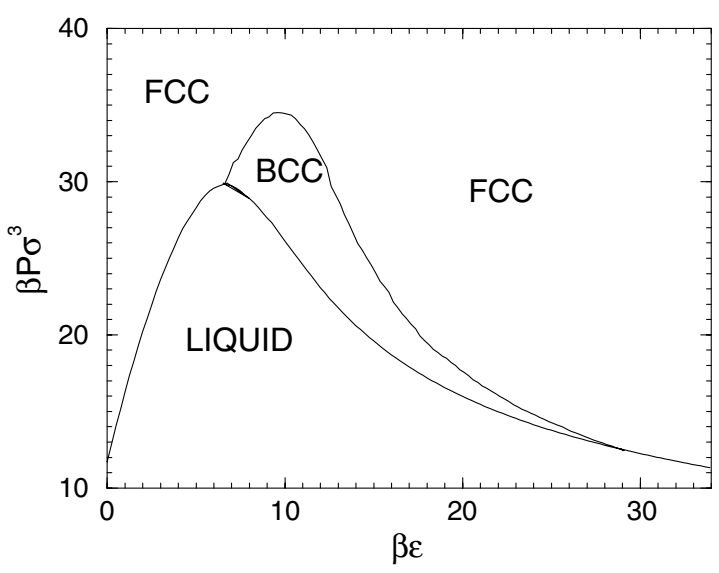

Figure 2. Calculated coexistence pressure from [6] for $\kappa=5$ as a function of the Yukawa repulsion $\beta \epsilon$.

The phase behaviour of the hard-core Yukawa potential has been calculated in detail by numerical simulation [6]. In these calculations the parameters $\kappa$ and $\beta \epsilon$ were varied independently. This cannot easily be done in experiments, as a variation in $\kappa$ will change $\epsilon$, unless some other parameter, such as the charge of the colloid, is varied at the same time.

The computed phase diagram of [6] shows a fluid-solid (bcc/fcc) and a solid-solid (bccfcc) coexistence line and it exhibits two fluid-bcc-fcc triple points (see figure 2). The main difference between the phase diagram of the hard-core Yukawa model and that of the pure (i.e. point-particle) Yukawa potential [7] is the presence of the second triple point. This triple point sets a lower limit for the strength of the Yukawa interaction for which a bcc phase exists.

Extensive crystallization experiments have been performed on systems of uncharged, hardsphere colloids using time-resolved laser light scattering or microscopy [8-14]. For charged colloidal systems, there are fewer studies of this kind [1, 15]. More recently, Gasser et al [16] published a confocal microscopy study of homogeneous crystal nucleation in slightly charged hard-sphere colloids. In addition, an extensive light-scattering study of crystallization in more highly charged colloids has been performed by Schöpe [2, 17, 18].

In the present paper, we report a computer simulation study of crystal nucleation in a hard-core Yukawa system. In these simulations, we have varied both the amplitude of the Yukawa repulsion and the magnitude of the screening length and studied the effect of both parameters on crystal nucleation.

\section{Homogeneous nucleation}

Crystal nucleation is an activated process. This implies that it costs free energy to form small crystal nuclei in the supersaturated liquid. However, once a nucleus exceeds a critical size, its free energy will decrease as further growth of the crystal takes place. Classical nucleation theory (CNT) provides a simple, thermodynamic description of the free energy of a crystal nucleus. In CNT theory the free energy of a nucleus contains two terms, a bulk and a surface term. The bulk term takes care of the fact that the chemical potential of the solid phase is lower than that of the liquid phase. The gain in free energy if $N$ particles transform from the liquid to the solid phase is $N \Delta \mu$, where $\Delta \mu=\mu_{\text {liq }}-\mu_{\text {sol }}$ is the difference in chemical potential between the liquid and the solid phase. The surface term, $A \gamma$, describes the free 
energy required to create a liquid/solid interface of area $A$, where $\gamma$ is the interfacial free energy per unit area. For small nuclei the surface term dominates and the free energy of the nucleus increases as it grows. When the nucleus is at its critical size $n_{\text {crit }}$ the function goes through a maximum:

$$
\Delta G^{*}=\frac{16 \pi}{3} \gamma^{3} /\left(\rho_{s}|\Delta \mu|\right)^{2},
$$

where $\rho_{s}$ is the number density of the solid phase. For larger nuclei, the free energy goes down again because it is dominated by the (negative) bulk term $-\Delta \mu$. The steady-state nucleation rate per unit volume, $I$, is the product of the probability that a critical nucleus is formed, $P_{\text {crit }}=\exp \left[-\beta \Delta G^{*}\right]$, times a kinetic factor, $\Gamma$, which describes the rate with which a critical nucleus growths:

$$
I=\Gamma \exp \left[-\beta \Delta G^{*}\right] .
$$

In order to be able to compare our results with experiments, we express the crystallization rates in dimensionless form: $I^{*} \equiv I \sigma^{5} / D_{0}$, where $D_{0}$ is the self-diffusion coefficient at infinite dilution. Such an expression for the reduced rate follows logically if all distances are expressed in units of $\sigma$ and time is expressed in units of $\sigma^{2} / D_{0}$. In addition, we choose $k_{B} T$ as our unit of energy. Other choices are possible (e.g. $\epsilon$ ). However, the present choice facilitates the comparison with earlier hard-sphere results [19]. In the following we will always use reduced quantities and hence we omit the asterisk.

We performed calculations of the nucleation barrier, kinetic prefactor and nucleation rate as a function of supersaturation varying both parameters of the Yukawa potential $\beta \epsilon$ and $\kappa$. In [6], the phase diagram has been computed as a function of $\beta \epsilon$ for $\kappa=5$ and as a function of $\kappa$ for $\beta \epsilon=8$. We chose to study the same range of parameters. As was already mentioned above, in experiments, $\kappa$ and $\epsilon$ are usually varied simultaneously. Hence, in order to compare with experiments, we have to combine and interpolate data from both sets of simulations.

One aspect of particular interest is the effect of the fcc-bcc-liquid triple point on the nucleation pathway. Already in 1897, Ostwald [20] had formulated his famous 'step rule'. This rule states that the phase that nucleates from the melt need not be the most stable solid phase, but is rather the one that is closest in free energy to the metastable liquid phase. Stranski and Totomanow [21] re-examined this rule and argued that the nucleated phase is the one that has the lowest nucleation barrier. Alexander and McTague [22] extended the Landau freeenergy expansion to freezing transitions that are weakly first order and concluded from general symmetry considerations that, in three dimensions, formation of the body-centred cubic (bcc) nuclei is uniquely favoured for simple fluids. However, simulations by ten Wolde et al [23] showed that the situation is more subtle, at least for the Lennard-Jones system: the core of the critical nucleus has the same structure as the stable crystal phase (fcc), but the surface bears a structural resemblance to the bcc phase that, for Lennard-Jones systems, is not stable. In the present system, we can 'tune' the relative stability of the fcc and bcc solids by moving past the triple point. Hence, this is an ideal system in which to study nucleation of a metastable phase.

\section{Simulations}

For the calculation of the nucleation barrier we used a biased Monte Carlo method [19, 23]. With this method we can compute the equilibrium probability $P(n)$ for the formation of a cluster of size $n$. This probability is related to the free energy of a crystalline cluster consisting of $n$ particles: $\Delta G(n)=$ constant $-\ln [P(n)]$. We first computed the nucleation barrier at fixed $\kappa=5$ for four different values of the amplitude of the Yukawa repulsion $\beta \epsilon=2,6,8$ and 20. Increasing the contact value $\beta \epsilon$ of the Yukawa repulsion shifts the volume fraction of 
Table 1. Excess free energy per particle for the different bulk structures and the liquid state calculated via a thermodynamic integration in the limit of infinite number of particles [25]. The reference state for the free-energy calculation of the liquid was the hard-sphere fluid, and for the bulk solid structures we used an Einstein crystal. In some cases we also used the hard-sphere system as a reference state for the solid structures. We found that the solid free energies obtained via these two distinct routes agreed to within $\pm 0.005 k_{B} T$, which corresponds to our estimate of the statistical error in this calculation. The statistical accuracy of the computed free energy of the liquid is estimated to be $\pm 0.01 k_{B} T$. In the table, the values in brackets indicate the volume fraction at which the excess free energy was calculated. The calculated excess free energies for the fcc and the hcp structures can be compared directly, as they were calculated at the same pressure, whereas the others are not. The fcc-hcp free-energy difference is always smaller than $1 \times 10^{-2} k_{B} T$.

\begin{tabular}{lllll}
\hline & $f_{f c c}$ & $f_{\text {hcp }}$ & $f_{b c c}$ & $f_{\text {liquid }}$ \\
\hline$\beta \epsilon=2$ & 12.894 & 12.892 & - & 11.38 \\
$\kappa=5$ & $(0.5425)$ & $(0.5425)$ & - & $(0.5032)$ \\
$\beta \epsilon=6$ & 23.258 & 23.256 & 21.49 & 19.11 \\
$\kappa=5$ & $(0.5027)$ & $(0.5027)$ & $(0.4808)$ & $(0.4503)$ \\
$\beta \epsilon=8$ & 24.344 & 24.35 & 24.32 & 22.23 \\
$\kappa=5$ & $(0.4563)$ & $(0.4563)$ & $(0.4558)$ & $(0.4329)$ \\
$\beta \epsilon=20$ & 20.872 & 20.873 & 20.986 & 16.16 \\
$\kappa=5$ & $(0.2888)$ & $(0.2888)$ & $(0.2895)$ & $(0.2529)$ \\
$\beta \epsilon=8$ & 11.144 & 11.147 & 11.067 & 10.02 \\
$\kappa=10$ & $(0.4084)$ & $(0.4084)$ & $(0.4054)$ & $(0.3853)$ \\
$\beta \epsilon=8$ & 39.107 & 39.110 & - & 38.08 \\
$\kappa=3.33333$ & $(0.5168)$ & $(0.5168)$ & - & $(0.5055)$ \\
\hline
\end{tabular}

the liquid phase at freezing to lower values than the hard-sphere value $\eta=0.494$. In order to be able to interpret our numerical data on the free-energy barrier for crystal nucleation, we need an accurate estimate of the density, pressure and chemical potential of the liquid at freezing. The data of [6] were obtained using a (modified) Gibbs-Duhem integration method. While this technique is useful to estimate the location of solid-liquid coexistence curves, the computed coexistence data were not sufficiently accurate for the present purpose. We therefore computed the location of all coexistence points by direct free-energy calculation of the solid and liquid phases [24]. The results for the excess free energy per particle are summarized in table 1. From the computed free energies, we obtain estimates for the chemical potential at freezing that have an error of $\pm 0.01 k_{B} T$. We found the following values for the volume fraction of the liquid phase at freezing: $\eta=0.482,0.438,0.405$ and 0.262 for $\beta \epsilon=2,6,8$ and 20 , respectively (see table 2).

\subsection{Nucleation barriers}

In figure 3 we show the results for the barrier height as a function of supersaturation with respect to the stable solid phase (fcc). As the figure shows, the main effect of increasing the strength of the Yukawa repulsion is to lower the nucleation barrier at constant supersaturation $\Delta \mu$.

Note that the decrease of the height of the nucleation barrier is particularly strong when only a weak repulsion is added to the hard-core potential. In particular, switching on a repulsive Yukawa potential with a contact value of only $2 k_{B} T$ decreases the nucleation barrier by some $10 k_{B} T$. This implies that for real hard-sphere colloids, the presence of only a small amount of charge can enhance the nucleation rate at constant volume fraction by many orders of magnitude through two mechanisms. First of all, the charge increases the supersaturation 


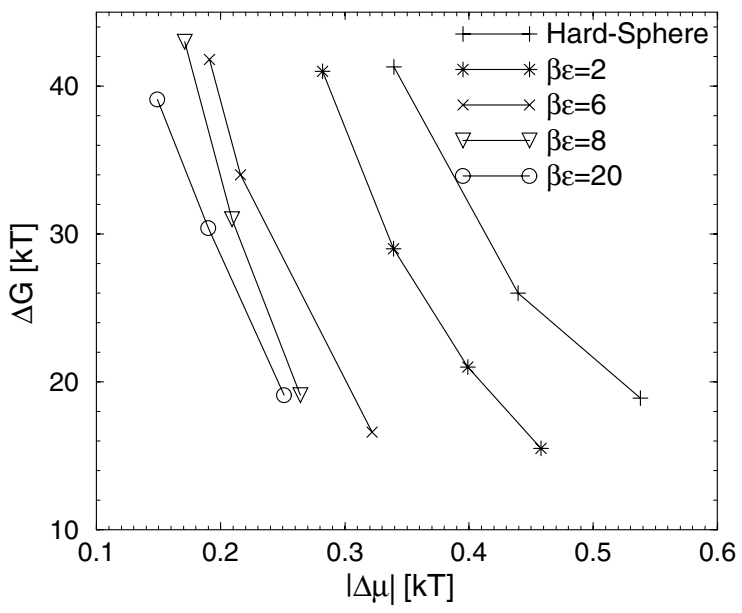

Figure 3. Calculated barrier heights of the hard-core Yukawa system with $\kappa=5$ and $\beta \epsilon=$ $2,6,8,20$ plotted as a function of supersaturation $\Delta \mu$ of the liquid phase with respect to the stable fcc phase.

Table 2. Summary of the data for the calculations with the repulsive hard-core Yukawa potential. Here $P$ is the pressure and $\eta_{l i q}, \eta_{f c c}, \eta_{b c c}$ the corresponding volume fractions of the liquid, fcc phase and bcc phase. $\Delta \mu_{f c c}$ and $\Delta \mu_{b c c}$ are the differences in chemical potential between the liquid and the fcc/bcc phases. The $\Delta G^{*}$ are the measured crystallization barriers. $f_{n_{c r i t}}^{+} / D_{0}$ is the reduced rate of attachment of particles to the critical cluster. $I$ is the calculated reduced nucleation rate.

\begin{tabular}{llllllllll}
\hline & $P$ & $\eta_{\text {liq }}$ & $\eta_{f c c}$ & $\eta_{b c c}$ & $\Delta \mu_{f c c}$ & $\Delta \mu_{b c c}$ & $\Delta G^{*}$ & $f_{n_{c r i t}}^{+} / D_{0}$ & $\log _{10}(I)$ \\
\hline$\kappa=5$ & 25 & 0.5103 & 0.5420 & - & 0.28 & - & 41 & 46 & -19.1 \\
and & 26 & 0.5159 & 0.5484 & - & 0.34 & - & 29 & 84 & -13.5 \\
$\beta \epsilon=2$ & 27 & 0.5218 & 0.5551 & - & 0.40 & - & 21 & 6 & -11.1 \\
& 28 & 0.5257 & 0.5599 & - & 0.46 & - & 15.5 & 19 & -8.1 \\
$\kappa=5$ & 37 & 0.4714 & 0.4827 & 0.4808 & 0.19 & 0.15 & 48.1 & 202 & -19 \\
and & 38 & 0.4755 & 0.4864 & 0.4848 & 0.22 & 0.17 & 34 & 57 & -16.1 \\
$\beta \epsilon=6$ & 42 & 0.4903 & 0.5031 & 0.5004 & 0.32 & 0.25 & 16.6 & 52 & -8.3 \\
$\kappa=5$ & 38 & 0.4415 & 0.4487 & 0.4481 & 0.17 & 0.15 & 43 & 218 & -19.5 \\
and & 40 & 0.4491 & 0.4563 & 0.4558 & 0.21 & 0.19 & 31 & 200 & -14.3 \\
$\beta \epsilon=8$ & 43 & 0.4596 & 0.4671 & 0.4668 & 0.26 & 0.24 & 19.1 & 300 & -8.8 \\
$\kappa=5$ & 23 & 0.2859 & 0.2888 & 0.2895 & 0.15 & 0.14 & 39.1 & 167 & -18.2 \\
and & 25 & 0.2938 & 0.2973 & 0.2974 & 0.19 & 0.19 & 30.4 & 58 & -14.8 \\
$\beta \epsilon=20$ & 28 & 0.3048 & 0.3084 & 0.3083 & 0.25 & 0.25 & 19.1 & 53 & -9.7 \\
$\kappa=10$ & 18 & 0.3848 & 0.3978 & 0.3949 & 0.23 & 0.15 & 49 & 80 & -22.6 \\
and & 20 & 0.3955 & 0.4084 & 0.4054 & 0.32 & 0.21 & 26.5 & 44 & -13 \\
$\beta \epsilon=8$ & 22 & 0.4054 & 0.4180 & 0.4150 & 0.40 & 0.28 & 15.2 & 11 & -8.5 \\
$\kappa=3.33333$ & 57 & 0.4937 & 0.5042 & - & 0.24 & - & 31.5 & 205 & -14.4 \\
and & 59 & 0.4996 & 0.5106 & - & 0.28 & - & 22.5 & 81 & -10.8 \\
$\beta \epsilon=8$ & 61 & 0.5055 & 0.5168 & - & 0.33 & - & 15.8 & 80 & -7.7 \\
\hline
\end{tabular}

at constant density. This effect would shift the nucleation curve to lower densities. But, in addition, the charge lowers the nucleation barrier at constant supersaturation. Further increase of the strength of the Yukawa repulsion leads to some additional decrease of the nucleation barrier, but the effect seems to saturate for values of $\beta \epsilon$ between 8 and 20 . 


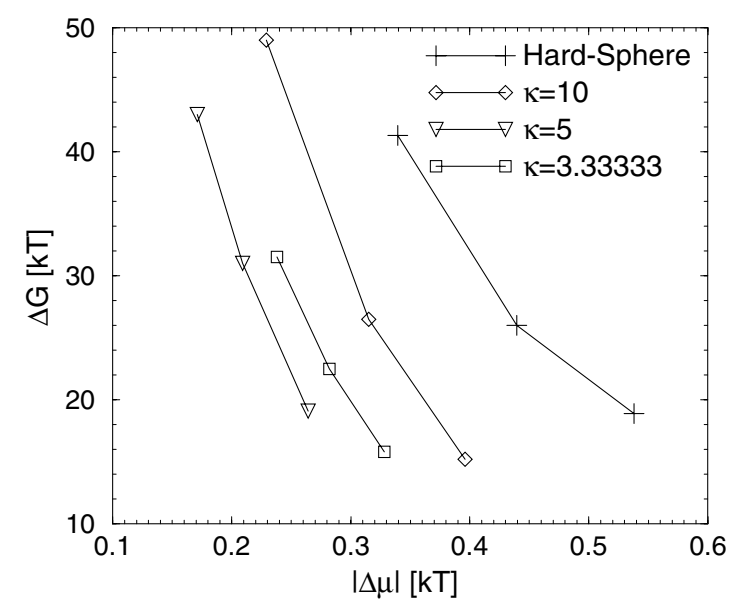

Figure 4. Calculated barrier heights of the hard-core Yukawa system with $\beta \epsilon=8$ and $\kappa=10,5,3.33333$ plotted as a function of supersaturation $\Delta \mu$ of the liquid phase with respect to the stable fcc phase.

Let us next consider the effect of the range of the repulsive potential on the nucleation barrier. We computed the height of the crystallization barrier for $\kappa=10,5$ and 3.33333 at a fixed contact value $\beta \epsilon=8$. In addition, we know the behaviour of the system in the hard-sphere limit $(\kappa=\infty)$. As $\kappa$ is decreased, the range of the potential grows. Initially (as $\kappa$ is decreased from $\infty$ to 10 ), the density at which the liquid freezes shifts from $\eta=0.494$ to 0.354 . Subsequently, the freezing density increases again. For $\kappa=5$, the volume fraction at freezing is $\eta=0.405$ and for $\kappa=3.333$, the liquid freezes at $\eta=0.456$. The variation of the crystallization barrier with $\kappa$ and $\Delta \mu$ is shown in figure 4 . The figure shows that increasing the range of the repulsive interaction, at constant supersaturation, initially has the effect of lowering the nucleation barrier. However, as $\kappa$ is decreased below 5, the nucleation barrier starts to increase again.

From the CNT expression for the height of the nucleation barrier, equation (2), we can estimate the corresponding values for the liquid/fcc interfacial free energy $\gamma_{f c c}$. In figure 5 we show the variation of the interfacial free energy with $\beta \epsilon$ at fixed $\kappa$. Figure 6 shows the variation of the interfacial free energy with $\kappa$ at fixed $\beta \epsilon$ for various values of the supersaturation $\Delta \mu$. The dependence of the interfacial free energy on the range of repulsion mirrors that of the nucleation barrier and is therefore non-monotonic. Coming from the hard-sphere limit, the interfacial free energy initially goes down, but for $\kappa<5$, it increases again.

In $[19,26]$ we found that, for hard spheres, the interfacial free energy $\gamma$ increases with supersaturation $\Delta \mu$. As can be seen in figure 6 , such behaviour is also observed in a system of charged colloids. In polydisperse hard-sphere systems [26], the increase of $\gamma$ with supersaturation could even result in a non-monotonic dependence of the nucleation barrier on supersaturation. In the present system, the interfacial free energy also increases with supersaturation, but the effect is not strong enough to result in a minimum in the nucleation barrier.

\subsection{Nucleation rates}

In order to calculate the absolute nucleation rate, equation (3), we need to evaluate the kinetic prefactor, which has the following form: $\Gamma=Z \rho_{l} f_{n_{\text {crit }}}^{+}\left(\sigma^{5} / D_{0}\right)$ [28]. Here $Z$ is 


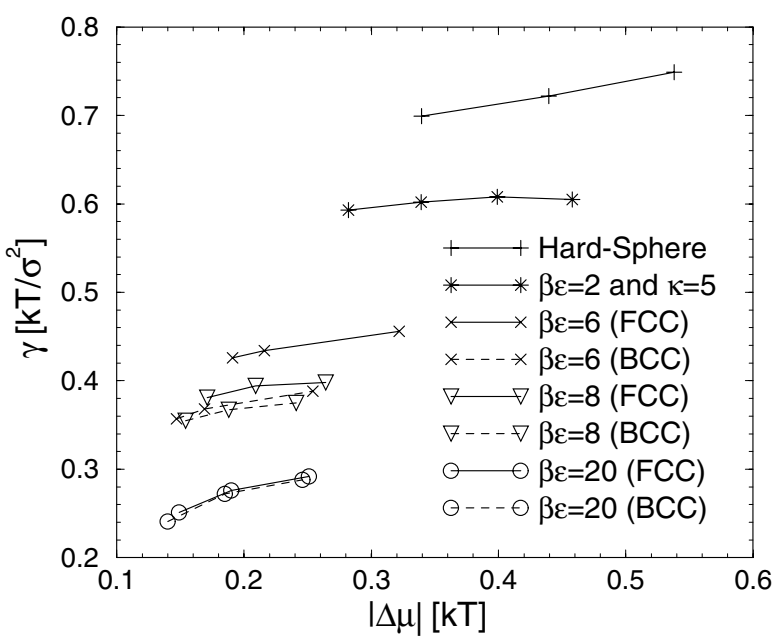

Figure 5. Interfacial free energy calculated from the barrier heights equation (2) for $\kappa=5$ and $\beta \epsilon=2,6,8,20$. The solid lines are the results assuming that the nuclei have a fcc structure, and the dashed lines are the results if the nuclei are bcc.

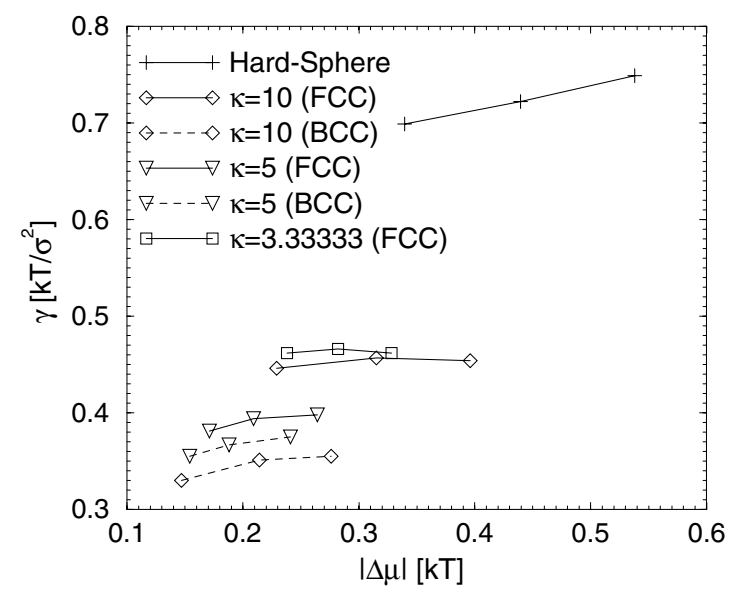

Figure 6. Interfacial free energy calculated from the barrier heights equation (2) for $\beta \epsilon=8$ and $\kappa=10,5,3.33333$. The solid lines are the results assuming that the nuclei have a fcc structure, and the dashed lines are the results if the nuclei are bcc.

the Zeldovich factor, $\rho_{l}$ the number density of the liquid phase and $f_{n_{\text {crit }}}^{+}$the rate of attachment of particles to the critical cluster. $D_{0}$ denotes the diffusion coefficient of the charged colloids at infinite dilution and $\sigma$ is the hard-core diameter of the Yukawa particles. The Zeldovich factor $Z=\left[\left|\Delta G^{\prime \prime}\left(n_{\text {crit }}\right)\right| /(2 \pi k T)\right]^{2}$ depends only on the second derivative of the nucleation barrier at its maximum. This information we obtain directly from our numerical results for $\Delta G(n)$. In order to compute $f_{n_{c r i t}}^{+} / D_{0}$, we used the kinetic Monte Carlo scheme of [29]. Basically, the method is a numerical scheme for solving the Smoluchowski equation. In doing this, we neglected the hydrodynamic interactions between the colloids. This drawback can be remedied by using the solution suggested by Medina-Noyola [30]. In this approach, the hydrodynamic interactions are taken into account through their effect on the short-time 


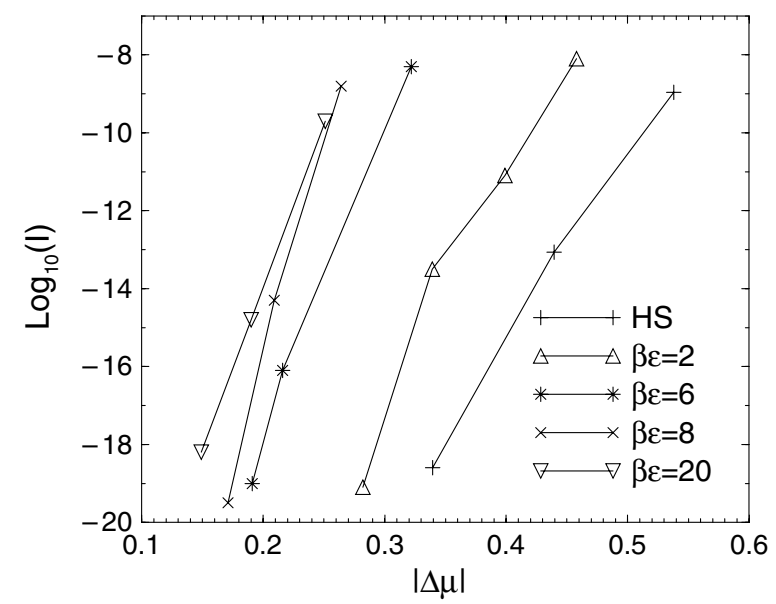

Figure 7. Dependence of the crystallization rates on the amplitude of the Yukawa repulsion $\beta \epsilon=2,6,8,20$ for $\kappa=5$ plotted as a function of supersaturation $\Delta \mu$ of the liquid with respect to the stable fcc phase.

self-diffusion coefficient. In practice, this means that we assume that, at short times, the mean squared displacement of a particle is not dominated by the infinite-dilution value of the diffusion coefficient $D_{0}$, but by the short-time self-diffusion coefficient $D_{S}^{S}$. The latter quantity differs from $D_{0}$ precisely because of hydrodynamic interactions. We therefore need to know the value of the ratio $D_{S}^{S} / D_{0}$ at the liquid density of interest. For hard-sphere suspensions, approximate expressions exist for the density dependence of $D_{S}^{S} / D_{0}$, e.g. $(1-\eta / 0.64)^{1.17}$ [31] As a test of this procedure, we used it to estimate the long-time self-diffusion coefficient of dense hard-sphere suspensions. The results agree within the statistical error with the available experimental data [32]. This gives us confidence that a similar approach can also be used to compute nucleation rates. However, the Medina-Noyola procedure is not directly applicable to the Yukawa system. To resolve this problem, we assumed that we could map the dynamics of the Yukawa system onto that of the hard-sphere system. To this end, we defined an effective packing fraction of the Yukawa system such that the packing fractions at freezing of the two systems were equal. This mapping is inspired by the observation of Löwen et al [33] that for many different colloidal systems, the ratio $D_{L}^{S} / D_{S}^{S}$ has a 'universal' value of 0.1 at freezing (here $D_{L}^{S}$ is the long-time self-diffusion constant). This rule suggests a dynamic 'corresponding-states principle' provided all densities are scaled to the freezing density. In fact, experiments by van Blaaderen et al [34] suggest that, for dense, charged colloids such a rescaling of the long-time self-diffusion coefficient is justified. While this approach is rather ad hoc, the systematic errors that it might induce are smaller than the random errors due to statistical inaccuracies in the determination of the height of the nucleation barrier.

3.2.1. Results. Our results for the computed nucleation rates are shown in figures 7 and 8 , where we plot the nucleation rate as a function of supersaturation. As the kinetic prefactor does not vary strongly with either supersaturation or interaction potential, the variation of the nucleation rate shown in figures 7 and 8 reflects the behaviour of the barrier height.

In order to compare the computed crystallization rates with the results of the confocal microscopy experiments of [16], we need to know the potential parameters that best characterize the experimental system that they used. From the fact that the suspensions studied by Gasser 


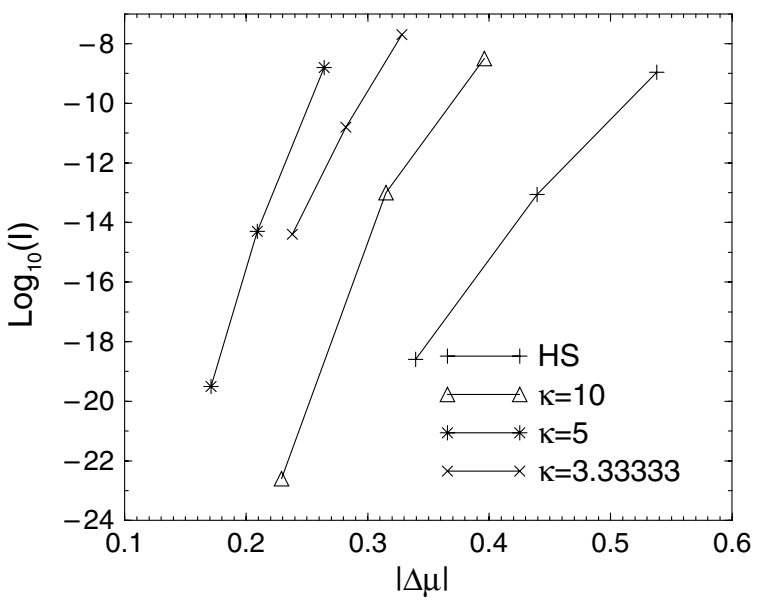

Figure 8. Dependence of the crystallization rates on the inverse screening length $\kappa a=$ $10,5,3.33333$ for $\beta \epsilon=8$ plotted as a function of supersaturation $\Delta \mu$ of the liquid with respect to the stable fcc phase.

et al freeze at a volume fraction $\eta=0.38$, it is clear that the colloidal particles used in these experiments are slightly charged. It is therefore natural to describe them by a Yukawa model that also has its freezing point at $\eta=0.38$. This condition is, however, not sufficient to fix the values of both $\kappa$ and $\epsilon$. For instance, if $\kappa=5$, then the observed freezing density can be reproduced by choosing $\beta \epsilon \sim 7$. Conversely, if we choose $\beta \epsilon=8$, then there are in fact two values of $\kappa$ that will reproduce the observed freezing density $(\kappa \sim 20$ and 6) [6].

In figure 9, we show a comparison of the nucleation rates reported in [16] with the simulation results for those $\kappa-\beta \epsilon$ combinations that yield a freezing point near $\eta=0.38$. As can be seen from the figure (and from the numbers collected in table 2), different $\kappa-\beta \epsilon$ combinations yield very different nucleation rates. However, the main effect of the variation of $\kappa$ and $\epsilon$ is to shift the nucleation curves horizontally: the slopes of the different curves are all rather similar. When we compare the computed nucleation rates with the experimental data, we note two things: first of all, the experimental rates tend to be (much) higher than the computed rates (Gasser et al find $-6.9 \leqslant \log [I] \leqslant-6.5$ for $\eta$ between 0.45 and 0.53 ). But, more importantly, the experiments suggest that the nucleation rate barely varies with volume fraction. This observation is hard to reconcile with the behaviour of any of the Yukawa models that we studied.

This discrepancy between experiment and simulation results suggests that it is incorrect to assume that the experimental system can be mapped onto a Yukawa model with densityindependent $\kappa$ and $\epsilon$. On the contrary, it is very likely that the effective potential parameters of weakly charged colloids in the absence of added salt depend strongly on concentration. In fact, recent experiments by Schöpe et al [17] clearly illustrate this effect: with increasing concentration, the effective potential of charged polystyrene spheres in dilute aqueous solution becomes increasingly hard-sphere-like. If we assume that the same phenomenon occurs in the more concentrated suspensions of [16], then experimental results for the nucleation rates at different densities should be compared with the numerical predictions that correspond to different effective Yukawa potentials.

As can be seen from figure 9 , the variation of the nucleation rate with density can be strongly reduced (and can possibly even become non-monotonic) if, as we expect, $\epsilon$ and $\kappa$ decrease with density. It is, however, not obvious that this effect is large enough to account for the apparent 


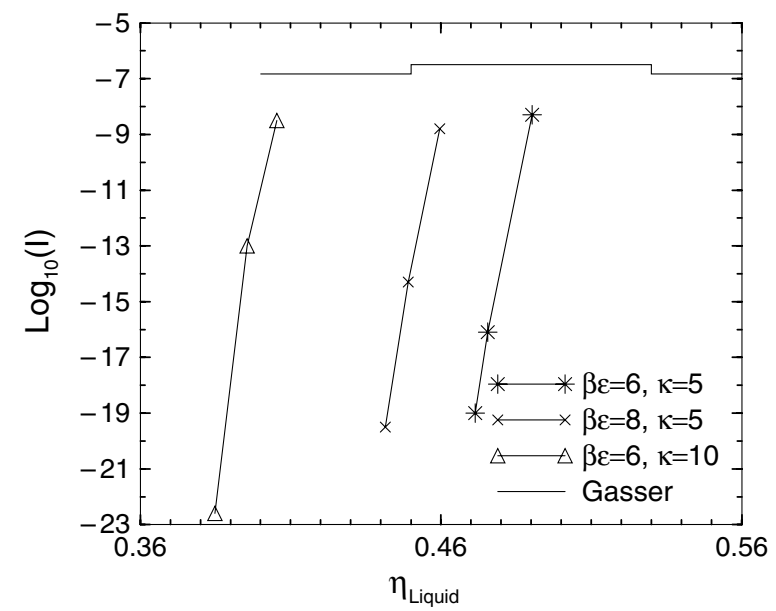

Figure 9. Comparison between the experimentally measured nucleation rates [16] and the simulation data. In the plot we added only the data sets which match the freezing density of the experimental system.

discrepancy between experiment and simulation. Clearly, a truly quantitative comparison between simulation and experiment requires better knowledge of the density dependence of the effective interaction between slightly charged colloidal spheres.

\subsection{Nucleation pathways}

The repulsive Yukawa system offers a unique opportunity to study the effect of metastable crystal phases on the pathway for crystal nucleation. The role of metastable phases in crystal nucleation was first pointed out by Ostwald [20] who, in 1897, formulated his 'step rule'. This rule states that the crystal phase that is nucleated from solution need not be the one that is thermodynamically most stable, but is the one that is closest in free energy to the fluid phase. Stranski and Totomanow [21] re-examined this rule and argued that the nucleated phase is the phase that has the lowest free-energy barrier of formation, rather than the phase that is globally stable under the conditions prevailing. More recently, Alexander and McTague [22] argued, on the basis of Landau theory, that in the early stages of crystal nucleation the formation of bcc crystallites should be favoured. Similar conclusions were subsequently reached by other groups $[35,36]$. In the Yukawa system, the fluid phase can coexist with either the fcc or the bcc phase, depending on the values of $\kappa$ and $\beta \epsilon$ (see figure 2). We can therefore study crystal nucleation both in the regime where the fcc phase is stable, and where the bcc phase is stable. If Ostwald's rule applied strictly, we should expect fcc nuclei to form where bcc crystals are stable, and conversely. On the other hand, if the Alexander-McTague scenario is correct, we should expect to find that bcc nuclei are always preferred. There is little point in testing the Stranski-Totomanow hypothesis as it amounts almost to a tautology ('the phase that nucleates fastest is the one with the lowest nucleation barrier').

To study the effect of metastable intermediates on crystallization, we analysed the structure of the (pre-)critical nucleus in different regions of the phase diagram shown in figure 2. As can be seen from figure 2, the pressure range region where the bcc phase is stable is rather narrow. For these pressures, the supersaturation of the fluid phase is small, and hence the nucleation barrier is very high. As a consequence, we could only study the formation of pre-critical 


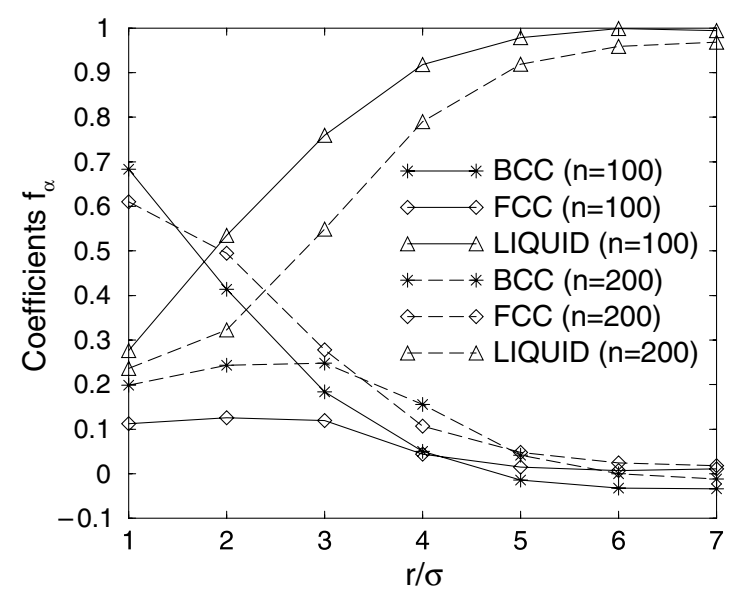

Figure 10. Structure analysis of two independent crystal nuclei of size $n=100$ and 200 from the simulations with parameters $\beta \epsilon=8$ and $\kappa=10$. The figure shows the results for the fit parameters for the local bond-order analysis as a function of the distance from the centre of mass of the nuclei. The core of the cluster of size $n=100$ has a clear bcc signature, where the cluster of size $n=200$ shows a clear fcc structure.

nuclei in this regime. In order to study the structure of the (pre-)critical nuclei, we used the local bond-order analysis proposed by ten Wolde et al [23]. In this analysis the local bondorder signature of a nucleus is decomposed into the signatures of the different bulk structures (liquid, fcc and bcc) using a linear least-squares fit. The values of the resulting coefficients $\left\{f_{\text {liq }}, f_{f c c}, f_{b c c}\right\}$ are a measure of the structure of the nucleus.

Our simulations show that the pre-critical nuclei always have a strong bcc signature. Only for larger (post-)critical nuclei well inside the fcc regime do we find a mixture of bcc and fcc signatures. In this sense, our simulations unambiguously support the prediction that nucleation into bcc nuclei is always uniquely favoured, even when the fcc phase is closer in free energy to the fluid phase.

Figure 10 shows the results of our cluster analysis for two distinct nuclei of size $n=100$ and 200. The picture shows the variation of the structural signature with the distance from the centre of mass of the nucleus. The results shown in this figure apply to the case of $\kappa=10$ and $\beta \epsilon=8$. This corresponds to the points in the phase diagram where the preference for the fcc structure is strongest. The core of the cluster of size $n=100$ has a clear bcc signature while the fcc phase does not seem to play a role. However, for the larger nuclei $(n=200)$ the core of the nuclei becomes fcc-like while the bcc phase seems to disappear. In this case the cluster transformation happened before it could reach critical size. This phase transition in the pre-critical nucleus allows us to quantify what value of the bcc-fluid interfacial free energy is needed in order to compensate for the difference in chemical potential of the two bulk structures. From our free-energy calculations, we deduce $\mu_{b c c}-\mu_{f c c}=0.082 \pm 0.005$. We used the CNT expression for the barrier height to estimate the fcc-liquid interfacial free energy: $\gamma_{f c c}=0.446$. The transformation from bcc to fcc nuclei occurred for $n \approx 100$. At that point, the gain in bulk free energy is $100 \times 0.082=8.2 k_{B} T$. This free-energy gain must be compensated by the increase in surface free energy as the crystallite transforms from bcc to fcc. To estimate this surface free energy, we need to know the radius of the crystal nucleus for $n=100$. If we assume that the nucleus is spherical and that the solid is effectively incompressible, we arrive at the estimate $\gamma_{b c c}=0.379$. 
We find such a pre-critical transformation from bcc to fcc for $\beta \epsilon=2$ with $\kappa=5$, and for $\beta \epsilon=8$ with $\kappa=10$ and 3.333 33. In all the other cases $(\beta \epsilon=6,8,20$ with $\kappa=5)$ even the critical nuclei had a strong bcc signature. This observation has implications for the interfacial free energies shown in figures 5 and 6. In these figures, we show interfacial free energies that were computed from the CNT expression for the barrier height, assuming that the nucleus had the same structure as the stable crystal phase. We now see that, in some cases, the critical nucleus has a metastable bcc structure. This affects the value for $\Delta \mu$ in the CNT expression, and hence our estimate for $\gamma$. In the cases where the critical nucleus has a bcc structure, we therefore also estimated the value of $\gamma_{b c c}$ from the height of the nucleation barrier. The results are also shown in figures 5 and 6.

Thus far we have not mentioned the possibility that the structure of the crystal nuclei could also be hexagonal closed packed (hcp) or a random stacking of the fcc and hcp domains (rhcp). In the case of hard spheres it is known that the free-energy difference between the stable fcc and hcp solid structure is very small $\left(\approx 10^{-3} k_{B} T\right)$ and therefore stacking faults are expected. Such stacking faults have been observed in experiments and computer simulations. In the case of charged spheres the situation is less clear. Some experiments indicate that the situation changes and there seems to be tendency for crystal nuclei to become more fcc-like [37]. Other experiments suggest that the structure of the cluster is still rhcp [16]. To resolve this question for the present model system, we first calculated the free-energy difference between the fcc and hcp solid, for all the different parameters of the model potential for which we performed rate calculations. It turns out that the free-energy difference per particle between the fcc and hcp structure was always smaller than $1 \times 10^{-2} k_{B} T$ (see table 1 ), which is about the limit of the accuracy that we had in our calculations. This means that thermal fluctuations of the order of a few $k_{B} T$ could easily transform clusters containing hundreds of particles from fcc to hcp, or generate intermediate stackings. To find out whether this really happens, we analysed the stacking of the (111) planes of ten nuclei with parameters $\beta \epsilon=8, \kappa=10$ and $\beta \epsilon=8, \kappa=3.333$ 33. In both cases, we do find stacking faults, but they seem to be less frequent than in the pure hard-sphere case. We stress, however, that these preliminary conclusions are based on the analysis of only a small number of crystallites.

\section{Acknowledgments}

All the numerical studies of crystallization that were performed in the 1970s focused on simple liquids ('argon') and liquid metals. The fact that it is now much more fruitful to study crystallization in colloidal systems, is due to the emergence of hard-core colloids as the experimental realization of a simple liquid. In this development, Peter Pusey (and his many collaborators) have played an absolutely crucial role. Therefore, without exaggeration, we can say that the present work would not have been possible but for the ground-breaking work of Peter Pusey.

We thank J Hoogenboom, A Yethiraj, A van Blaaderen, H J Schöpe and T Palberg for inspiring discussions and a critical reading of the manuscript. We also thank E J Meijer for permission to show figure 2. This work was supported by the division of Chemical Sciences (CW) of the Netherlands Organization for Scientific Research (NWO). The work of the FOM Institute is part of the research programme of FOM and is made possible by financial support from the Netherlands Organization for Scientific Research (NWO). An NCF grant of computer time on the TERAS supercomputer is gratefully acknowledged. 


\section{References}

[1] Dhont J K G, Smits C and Lekkerkerker H N W 1992 J. Colloid Interface Sci. 152386

[2] Schöpe H J and Palberg T 2001 Prog. Colloid Polym. Sci. 11882

[3] Verweij E J W and Overbeek J Th G 1948 Theory of the Stability of Lyophilic Colloids (New York: Elsevier)

[4] Alexander S et al 1984 J. Chem. Phys. 805776

[5] Bitzer F, Palberg T, Löwen H, Simon R and Leiderer P 1994 Phys. Rev. E 502821

[6] El Azhar F, Baus M, Ryckaert J P and Meijer E J 2000 J. Chem. Phys. 1125121

[7] Meijer E J and Frenkel D 1991 J. Chem. Phys. 942269

[8] Schätzel K and Ackerson B J 1993 Phys. Rev. E 483766

[9] Harland J L and van Megen W 1993 Phys. Rev. E 483766

[10] Cheng Z 1998 Thesis Princeton University, NJ

[11] Heymann A, Stipp A, Sinn C and Palberg T 1998 J. Colloid Interface Sci. 206119

[12] Haddon M S, Haddon S B and Poon W C K 2001 J. Phys.: Condens. Matter 13553

[13] Sinn C, Heymann A, Stipp A and Palberg T 2001 Prog. Colloid Polym. Sci. 118266

[14] Palberg T 1999 J. Phys.: Condens. Matter 11323

[15] Okubo T, Okada S and Tsuchida A 1997 J. Colloid Interface Sci. 189337

Okubo T and Okada S 1997 J. Colloid Interface Sci. 192490

Okubo T, Tsuchida A and Kato T 1999 Colloid Polym. Sci. 277191

[16] Gasser U, Weeks E R, Schofield A, Pusey P N and Weitz D A 2001 Science 292258

[17] Schöpe H J 2000 Dissertation Johannes Gutenberg Universität Mainz

[18] Schöpe H J and Palberg T 2002 J. Noncryst. Mater. at press

[19] Auer S and Frenkel D 2001 Nature 4091020

[20] Ostwald W 1897 Z. Phys. Chem. 22289

[21] Stranski I N and Totomanow D 1933 Z. Phys. Chem. 163399

[22] Alexander S and McTague J P 1978 Phys. Rev. Lett. 41702

[23] ten Wolde P R, Ruiz-Montero M J and Frenkel D 1995 Phys. Rev. Lett. 752714

[24] Frenkel D and Smit B 2002 Understanding Molecular Simulation: from Algorithms to Applications 2nd edn (New York: Academic)

[25] Polson J M, Trizac E, Pronk S and Frenkel D 2000 J. Chem. Phys. 1125339

[26] Auer S and Frenkel D 2001 Nature 413711

[27] Pusey P N and van Megen W 1986 Nature 320340

[28] Kelton K F 1991 Solid State Physics vol 45 (New York: Academic)

[29] Hinsen K and Cichocki B 1990 Physica A 166473

[30] Medina-Noyola M 1988 Phys. Rev. Lett. 602705

[31] Van Duijneveldt J S and Lekkerkerker H N W 1995 Science and Technology of Crystal Growth ed J P van der Eerden and O S L Bruinsma (Dordrecht: Kluwer Academic)

[32] Auer S and Frenkel D 2002 in preparation

[33] Löwen H, Palberg T and Simon R 1993 Phys. Rev. Lett. 701557

[34] van Blaaderen A, Peetermans J, Maret G and Dhont J K G 1992 J. Chem. Phys. 964591

[35] Groh B and Mulder B 1999 Phys. Rev. E 595613

[36] Klein W and Leyvraz F 1986 Phys. Lett. 572845

[37] Hoogenboom J P, Derke D, Verger P and van Blaaderen A 2002 in preparation 